\title{
Parity violation in radiative neutron capture on deuteron
}

\author{
Young-Ho Song* \\ University of South Carolina, Columbia, SC 29208, USA \\ E-mail: song25@mailbox.sc.edu
}

\section{Vladimir Gudkov}

University of South Carolina, Columbia, SC 29208, USA

E-mail: gudkovesc.edu

\section{Rimantas Lazauskas}

IPHC, IN2P3-CNRS/Université Louis Pasteur, France

E-mail: rimantas.lazauskaseires.in2p3.fr

\begin{abstract}
Parity violating (PV) effects in neutron-deuteron radiative capture process are computed for the case of Desplanques, Donoghue, and Holstein (DDH) and effective field theory (EFT) PV weak potential models. Wave functions of the system are computed by solving three-body Faddeev equations in configuration space for both realistic strong potential models and PV weak potential models. The relations between physical observables and low-energy constants are presented, and dependencies of the calculated PV effects on strong and weak potentials are discussed.
\end{abstract}

The 7th International Workshop on Chiral Dynamics,

August 6 -10, 2012

Jefferson Lab, Newport News, Virginia, USA

${ }^{*}$ Speaker. 


\section{Introduction}

The importance of parity violating (PV) effects in the standard model made many nuclear PV effects be measured and calculated during the last several years. Lately it became clear $[1,2]$ that it is rather difficult to describe the available experimental data with the same set of weak nucleon coupling constants using the traditional DDH [3] weak meson exchange potential.

As a possible solution for this problem, a new approach, based on the effective field theory (EFT), has been introduced to parameterize PV effects in a model independent way $[1,2,4]$. To avoid theoretical uncertainty from heavy nuclear system, it is desirable to use few nucleon system to determine low energy constants (LECs). Unfortunately, the number of experimentally measured (and independent in terms of unknown LECs) PV effects in two body systems is not enough to constrain all LECs $[5,6,7,8]$. In order to determine these constants, it is necessary to include also the data obtained on heavier nuclear systems.

In addition, the study of PV effects in heavier nuclei is neccesarry because these effects might be essentially enhanced $[9,10,11]$ in many body systems. To verify the possible issues related to the application of the DDH description of PV effects in nuclei and the possibility of systematic calculations of PV effects in nuclei using the EFT approach, it is desirable to start from the calculations of PV effects in the simplest nuclear systems, such as neutron-deuteron (n-d) compound. PV effects for elastic n-d scattering have been calculated recently $[12,13]$ using both the DDH and the EFT approaches. However, before extending these techniques to many-body nuclear systems, it is important to consider inelastic processes which are usually more sensitive to short range interactions.

With this aim, we present a comprehensive analysis of PV effects in neutron-deuteron radiative capture using weak potential of the DDH-type, as well as weak potentials obtained in pionless and pionful EFT with realistic strong potential models in a "hybrid" method.

\section{Formalism}

We consider three parity violating observables in the radiative neutron capture on deuterons $\left(n+d \rightarrow{ }^{3} H+\gamma\right)$ at thermal neutron energy: circular polarization of emitted photons $\left(P^{\gamma}\right)$, asymmetry of photons in relation to neutron polarization $\left(a_{n}^{\gamma}\right)$, and asymmetry of photons in relation to deuteron polarization $\left(A_{d}^{\gamma}\right)$. For low energy neutrons, these PV effects appear as interference between parity conserving magnetic dipole $(M 1)$ and parity violating electric dipole $(E 1)$ transition matrix elements. M1 and E1 amplitudes are defined as reduced matrix elements of the electromagnetic multipole operators. We define real-valued $\widetilde{\mathscr{M}}_{J}$ and $\widetilde{\mathscr{E}}_{J}$ matrix elements as

$$
M 1_{J}=i \frac{\omega /\left(2 m_{N}\right)}{\sqrt{6 \pi} \sqrt{4 \pi}} \widetilde{\mathscr{M}_{J}}, \quad E 1_{J}=-i \frac{\omega}{\sqrt{6 \pi}} \widetilde{\mathscr{E}}_{J}
$$

where $J$ represent total angular momentum of scattering wave.

The results for parity conserving $M 1$ amplitudes for radiative thermal neutron capture on deuteron using "hybrid" method up to $N^{3} L O$ in chiral order counting is reported in papers $[14,15]$. We adopted the result of [14], which corresponds to the total neutron-deuteron radiative capture cross section as $\sigma_{t o t}=0.49(1) \mathrm{mb}$. The result of [14] is in good agreement with experimental measurement and shows small model dependence. As for parity violating $E 1$ amplitude at the leading 
order, we use only the E1 charge operator from Siegert's theorem and thus only parity violation and potential model dependence occurs in the wave function.

The parity conserving and parity violating component of three-body wave function are obtained by solving Faddeev equations in a configuration space [16] while treating parity violating wave function $\psi^{-}$and potential $V_{i j}^{P V}$ as perturbations to those of parity conserving ones,

$$
\begin{aligned}
& \left(E-H_{0}-V_{i j}^{P C}\right) \psi_{k}^{+}=V_{i j}^{P C}\left(\psi_{i}^{+}+\psi_{j}^{+}\right), \\
& \left(E-H_{0}-V_{i j}^{P C}\right) \psi_{k}^{-}=V_{i j}^{P C}\left(\psi_{i}^{-}+\psi_{j}^{-}\right)+V_{i j}^{P V}\left(\psi_{i}^{+}+\psi_{j}^{+}+\psi_{k}^{+}\right)
\end{aligned}
$$

where $\psi_{k}=\psi_{i j, k}$ is so called Faddeev component. To solve these equations numerically, we use our standard procedure with generalization for three-nucleon force, as is described in detail in [17].

For a strong (parity conserving) part of the Hamiltonian, we choose a set of realistic models of nucleon-nucleon interactions, namely: Argonne v18 potential (AV18) [18], INOY potential [19], Reid soft-core potential (Reid) and Nijmegen potential(NijmII) [20]. Also, we have performed calculations for the AV18 potential in conjunction with the Urbana IX three-nucleon force potential [21] (denoted as AV18+UIX).

We considered three types of parity violating weak potentials: the standard DDH potential with meson exchange nucleon-nucleon interactions [3], and for two different choices of the EFT potentials [1], which are derived from pionless and pionful EFT Lagrangian. All these potentials can be expanded in terms of $O_{i j}^{(n)}$ operators [12] as

$$
v_{i j}^{\alpha}=\sum_{n} c_{n}^{\alpha} O_{i j}^{(n)}, \quad \alpha=\mathrm{DDH}, \text { pionless EFT or pionful EFT }
$$

where Operators $O_{i j}^{(n)}$ are written as products of iso-spin, spin operators and (anti-)commutator of momentum and scalar function, $\left[p_{i j}, f_{n}\left(r_{i j}\right)\right]_{ \pm}$. Explicit forms for the operators $O_{i j}^{(n)}$ and the corresponding parameters $c_{n}^{\alpha}$, listed in [13]. All potentials share the same operator forms, but differ in the magnitude of LECs and the form of scalar function, $f_{n}\left(r_{i j}\right)$. We typically chose the Yukawa type scalar function,

$$
f_{\mu}(r)=\frac{1}{4 \pi r} e^{-\mu r}
$$

for both DDH potential and EFT short range potential by taking $\mu$ as either meson mass or cutoff respectively.

Our approach could be considered as a "hybrid" method in the sense of using potential models derived from the Lagrangian of Effective theory, similar to the "hybrid" approach in the line of Weinberg's scheme [22]. The same approach was already applied to the calculations of parity violating [13] and time reversal violating [23, 24] effects in elastic $n-d$ scattering.

\section{Results and Discussions}

The results obtained with the DDH potential are in a reasonably good agreement with the previous calculations [25], considering the difference in wave functions, and give us the opportunity 

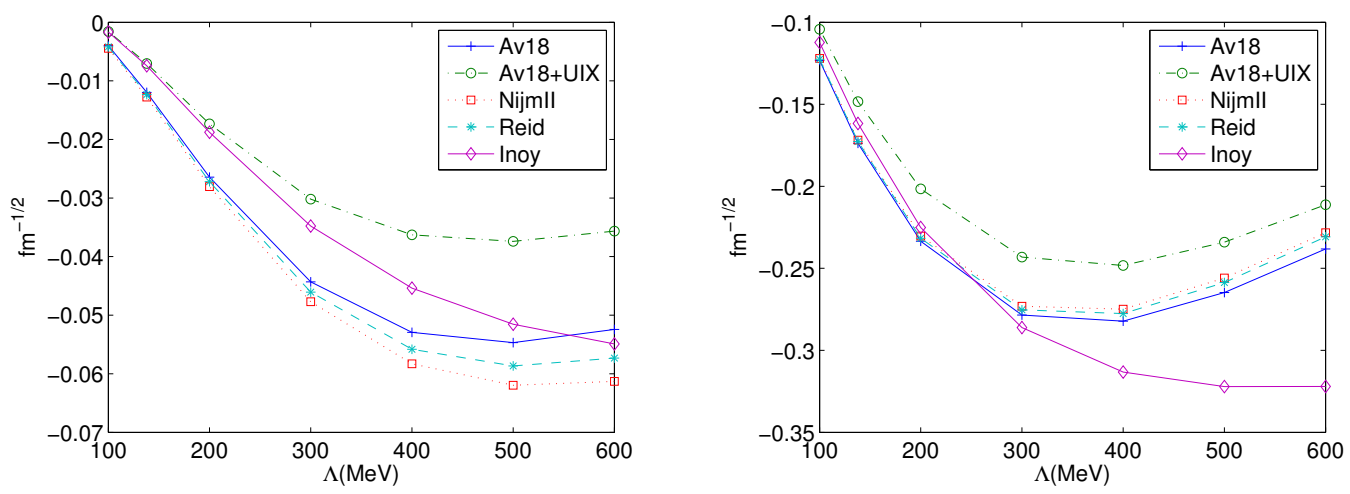

Figure 1: Cutoff and strong model dependencies of the amplitudes for $\pi$ EFT calculated with AV18, AV18+UIX, Nijmegen-II, INOY, and Reid strong potentials. The first graph shows $\mu^{2} \widetilde{\mathscr{E}}_{\frac{3}{2}(+)}$ for operator 1 and the second graph shows $\mu^{2} \widetilde{\mathscr{E}}_{\frac{3}{2}(+)}$ for operator 9 in $f m^{-\frac{1}{2}}$ units. The multiplier $\mu^{2}$ is used to absorb artificial cutoff dependence of $c_{n}$ coefficients. ( + ) represents the positive parity of $n-d$ wave function.

to estimate the values of all PV effects in terms of PV meson-nucleon coupling constants $h$ as

$$
\begin{aligned}
a_{n} & =0.42 h_{\pi}^{1}-0.17 h_{\rho}^{0}+0.085 h_{\rho}^{1}+0.008 h_{\rho}^{2}-0.238 h_{\omega}^{0}+0.086 h_{\omega}^{1}-0.010 h_{\rho}^{\prime 1}=4.11 \times 10^{-7}, \\
P_{\gamma} & =-1.05 h_{\pi}^{1}+0.19 h_{\rho}^{0}-0.096 h_{\rho}^{1}-0.018 h_{\rho}^{2}+0.28 h_{\omega}^{0}-0.046 h_{\omega}^{1}+0.023 h_{\rho}^{\prime 1}=-7.31 \times 10^{-7}, \\
A_{d}^{\gamma} & =-1.51 h_{\pi}^{1}+0.17 h_{\rho}^{0}-0.083 h_{\rho}^{1}-0.024 h_{\rho}^{2}+0.024 h_{\omega}^{0}+0.013 h_{\omega}^{1}+0.032 h_{\rho}^{\prime 1}=-9.05 \times 10^{-7} .
\end{aligned}
$$

The coefficients in these expressions are obtained using strong AV18+UIX and weak DDH potentials, while the final values of PV observables are given for the "best" values of the DDH coupling constants. Local strong two-body potential models (AV18, Reid, and NijmII) and threebody potential models (AV18+UIX) with "best" values of DDH coupling constants gives small model dependence in observables $a_{n}=(4.11 \div 4.74) \times 10^{-7}, P_{\gamma}=-(7.30 \div 8.45) \times 10^{-7}, A_{d}=$ $-(9.04 \div 10.5) \times 10^{-7}$. It should be noted that, however, the above results are specific combination of amplitudes for one set of DDH coupling constants. $P_{\gamma}$ and $A_{d}$ for different choice of LECs values and individual matrix elements for heavy meson exchange shows sensitivity to the strong potential model and the inclusion of 3-body interaction.

The PV effects in weak pionless EFT potentials with the same AV18+UIX strong potential and choice of $\mu=138 \mathrm{MeV}$ can be summarized as

$$
\begin{aligned}
& a_{n}=\frac{2 m_{N}}{\Lambda_{\chi}^{3}}\left(0.0217 C_{6}^{\pi t}-0.0794\left(C_{2}^{\pi t}+C_{4}^{\pi t}\right)+0.0562 C_{5}^{\pi t}-0.208 C_{1}^{\pi t}+0.0762 \widetilde{C}_{1}^{\pi t}\right), \\
& P_{\gamma}=\frac{2 m_{N}}{\Lambda_{\chi}^{3}}\left(-0.0552 C_{6}^{\pi t}+0.0655\left(C_{2}^{\pi t}+C_{4}^{\pi t}\right)+0.1192 C_{5}^{\pi t}-0.2060 C_{1}^{\pi t}-0.0858 \widetilde{C}_{1}^{\pi t}\right), \\
& A_{d}=\frac{2 m_{N}}{\Lambda_{\chi}^{3}}\left(-0.0793 C_{6}^{\pi t}+0.0316\left(C_{2}^{\pi t}+C_{4}^{\pi t}\right)+0.1602 C_{5}^{\pi t}-0.1516 C_{1}^{\pi t}-0.0734 \widetilde{C}_{1}^{\pi t}\right) .
\end{aligned}
$$

Pionful EFT results and tables of results with detailed explanation can be found in [26].

In the "hybrid" method which uses phenomenological potential models, it is important to check the model and cutoff dependence. The Fig.1 shows the cutoff $\Lambda$ and strong potential model 
dependence of E1 amplitudes in case of operator 1 and 9 of pionless EFT potential. Because we used the same scalar function for both DDH and pionless EFT, choice of mass scale as the meson mass corresponds to DDH results. At low mass scale, amplitudes show small potential model dependence. However, at heavy meson mass scale, the large strong potential model dependence is observed. Large model dependence of PV amplitudes at heavy mass scale implies that matrix elements related to $n-d$ radiative capture process are sensitive to these short range interactions. This sensitivity to a short range dynamics is new phenomenon observed in radiative $n$ - $d$ capture and may be partially responsible for the existing discrepancy [27] in the analysis of PV effects in contrast to the case of parity violation in elastic n-d scattering, where PV matrix elements are practically insensitive [13] to the choice of strong potentials.

For the case of the DDH approach, the observed model dependence indicates intrinsic difficulty in the description of nuclear PV effects. Thus, the DDH approach could be a reasonable approach for the parametrization and for the analysis of PV effects only if exactly the same strong and weak potentials are used in calculating all PV observables in all nuclei. However, the existing calculations of nuclear PV effects have been done using different potentials; therefore, strictly speaking, one cannot compare the existing results of these calculations among themselves. Further, most of the existing calculations do not include three body interactions which is shown to be important.

On the other hand, from the point of view of the pionless EFT, the reasonable cutoff mass scale cannot exceed the value of a pion mass, where the dependence on strong interaction potential is small. Since the cutoff in the EFT could be considered as a measure of our knowledge of short range physics, one can expect a smaller model dependence when the cutoff parameter is low. Thus the result implies the small model dependence is achievable even in "hybrid" approach calculation.

\section{Conclusion}

PV effects in neutron-deuteron radiative capture are calculated for DDH-type and EFT-type, pionless and pionful, weak-interaction potentials. The three-body problem was solved using Faddeev equations in configuration space, as well as by varying the strong interaction part of the Hamiltonian. A number of different realistic strong potentials have been tested, including the AV18 nucleon-nucleon interaction in conjunction with the UIX three-nucleon force. The analysis of the obtained results shows that the values of PV amplitudes depend on the choice of both weak and strong interaction models. In order to obtain model independent EFT predictions for PV observables, one should perform all calculations in a self-consistent way [28]. However, we demonstrated that this dependence has the expected behavior in the framework of the standard pionless and pionful EFT approaches even in the "hybrid" approach. Therefore, this dependence is expected to be absorbed by the LECs both in the "hybrid" approach and in the full EFT calculations.

ACKNOWLEDGMENTS: This work was supported by Department of Energy Grant No.DEFG02-09ER41621 and NSFGrant No. PHY-0758114. This work was granted access to the HPC resources of IDRIS under the allocation 2009-i2009056006 made by GENCI (Grand Equipement National de Calcul Intensif). We thank the staff members of the IDRIS for their constant help. 


\section{References}

[1] S.-L. Zhu et al. , Nucl. Phys.A748, 435 (2005).

[2] M. J.Ramsey-Musolf and S. A. Page, Ann. Rev. Nucl. Part. Sci.,56,52(2006).

[3] B. Desplanques, J. F. Donoghue, and B. R. Holstein, Ann. Phys. (NY) 124, 449 (1980).

[4] C. -P. Liu, Phys. Rev. C75, 065501 (2007).

[5] L. Girlanda, Phys. Rev. C77, 067001 (2008).

[6] D. R. Phillips, M. R. Schindler, and R. P. Springer, Nucl. Phys.A822, 1 (2009).

[7] J. W. Shin, S. Ando, and C. H. Hyun, Phys. Rev. C81, 055501(2010).

[8] M. R. Schindler and R. P. Springer, Nucl. Phys.A846, 51 (2010).

[9] O. P. Sushkov andV.V. Flambaum, Sov. Phys.Usp. 25, 1 (1982).

[10] V. E. Bunakov and V. P. Gudkov, Nucl. Phys. A 401, 93 (1983).

[11] V. P. Gudkov, Phys. Rep. 212, 77 (1992).

[12] R. Schiavilla, M. Viviani, L. Girlanda, A. Kievsky, and L. E. Marcucci, Phys. Rev. C 78, 014002 (2008).

[13] Y.-H. Song, R. Lazauskas, and V. Gudkov, Phys. Rev. C 83, 015501 (2011).

[14] Y.-H. Song, R. Lazauskas, and T.-S. Park, Phys. Rev.C 79, 064002 (2009).

[15] S. Pastore, L. Girlanda, R. Schiavilla, M. Viviani, and R. B. Wiringa, Phys. Rev. C 80, 034004 (2009).

[16] L. D. Faddeev, Sov. Phys. JETP 12, 1014 (1961).

[17] R. Lazauskas, Few-Body Systems 46, 37 (2009).

[18] R. B. Wiringa, V. G. J. Stoks, and R. Schiavilla, Phys. Rev. C 51, 38 (1995).

[19] P. Doleschall, I. Borbely, Z. Papp, and W. Plessas, Phys. Rev. C 67, 064005 (2003).

[20] V. G. J. Stoks, R. A. M. Klomp, C. P. F. Terheggen, and J. J. de Swart, Phys. Rev. C 49, 2950 (1994).

[21] B. S. Pudliner, V. R. Pandharipande, J. Carlson, and R. B. Wiringa, Phys. Rev. Lett. 74, 4396 (1995).

[22] S. Weinberg, Phys. Lett. B 251, 288 (1990); Nucl. Phys. B 363, 3 (1991).

[23] Y.-H. Song, R. Lazauskas, and V. Gudkov, Phys. Rev. C 83, 065503 (2011).

[24] Y.-H. Song, R. Lazauskas, and V. Gudkov, Phys. Rev. C 84, 025501 (2011).

[25] A. Moskalev, Sov. J. Nucl. Phys. 9, 99 (1969); E. H. E. Hadjimichael and V. Newton, Nucl. Phys. A 228, 1 (1974); B. H. McKellar, Phys. Rev. C 9, 1790 (1974); B. Desplanques and J. Benayoun, Nucl. Phys.A458, 689 (1986).

[26] Y.-H. Song, R. Lazauskas, and V. Gudkov, Phys. Rev. C 86, 045502 (2012).

[27] B. R. Holstein, Fizika B 14, 165 (2005). ; Eur. Phys. J.A 41, 279 (2009).

[28] V. Gudkov and Y.-H. Song, Phys. Rev. C 82, 028502 (2010).

[29] L. Girlanda et al. ., Phys. Rev. Lett. 105, 232502 (2010). 